letzterer Hinsicht fehlt jeder Anhalt dafür, wie weit sie von der Wahrheit entfernt sind.

Selbst wenn es gelänge, die bleibende Magnesia (Chlorid und Sulfat zusammen) im Flußwasser zu bestimmen, so ist es nicht erlaubt, aus dieser Summe auf die entsprechenden Carnallitmengen rückzuschließen, weil in den Flü:sen in der Regel viel mehr Magnesiumsulfat im Vergleich zum Chlorid vorhanden ist, als dem Verhältnis im Carnallit entspricht. Das trifft besonders zu für die Flüsse im Wesergebiet.

Nach dem Verfahren von P r e c h t findet man hingegen das mit den Endlaugen den Flüssen zugeführte Chlor magnesium einschließlich dem Chlorcalcium aus den Ammoniaksodafabriken mit hinreichender Genauigkeit, um die entsprechenden Verarbeitungsmengen an Carnallit berechnen zu können. Der auf die Kaliwerke allein entfallende Betrag ergibt sich nach Abzug der anderweitig für die Sodafabriken ermittelten, verhältnismäßig kleinen und im Gegensatz zur Kaliverarbeitung ziemlich gleichbleibenden Menge.

Will man den Zustand des Flußwassers hinsichtlich seiner Versalzung durch Chlormagnesium und das ähnlich wirkende Chlorcalcium erfassen, so liefert das $\mathrm{Prech}$ t verfahren unmittelbar den zutreffenden Ausdruck. Die Ermittlung des mit ähnlichen Wirkungen behafteten Magnesiumsulfats (Bittersalz) bleibt der besonderen Bestimmung im Rückstand vom Alkoholauszug vorbehalten.

Der Gehalt des Flußwassers an den drei bezeichneten Salzen begrenzt die Aufnahmefähigkeit weiterer Abwassermengen mit den gleichen Salzen, also namentlich der Kaliabwässer.

Über den etwaigen Gleichgewichtszustand der Salze im Wasser sagt das P r e c h t sche Verfahren ebensowenig aus wie das N o l I sche. Seiner Kenntnis bedarf es aber nicht, da die Wirkungen der Salze doch nur durch die dem Versuchswasser hinzugefügten Mengen und nicht etwa nach dem unbekannten Grad der Umsetzung und Ionenspaltung beurteilt werden können.

[A. 132]

\section{Studien über Sulfite, Thiosulfate und Polythionate $\mathrm{II}^{1}$ \%.}

\author{
Von Dr.-Ing. A. SANDER.
}

(Eingeg. 3./11. 1915.)

(Mitteilung aus dem chemisch-technischen und elektrochemischen Institut der Technischen Hochschule zu Darinstadt.)

\section{Einwirkung von Quecksilberchlorid auf schweflige Säure, Sulfite, Thiosulfate und Polythionate.}

(2. Mitteilung.)

In meiner früheren Veröffentlichung unter dieser Überschrift ${ }^{2}$ ) habe ich bereits darauf hingewiesen, daß die Umsetzung von Quecksilberchlorid mit Sulfiten in anderer Weise verläuft, als von $\mathrm{F}$ eld seinerzeit angenommen wurde. Während Feld nämlich der Meinung war, daß Natriumsulfit mit Quecksilberchlorid überhaupt nicht reagiert ${ }^{3}$ ), konnte ich zeigen, daß diese beiden Salze in folgender Weise aufeinander einwirken:

$$
\mathrm{HgCl}_{2}+2 \mathrm{Na}_{2} \mathrm{SO}_{3}=\mathrm{Hg} \underset{\mathrm{SO}_{3} \mathrm{Na}}{-\mathrm{SO}_{3} \mathrm{Na}}+2 \mathrm{NaCl} \text {. }
$$

Es entsteht also eine komplexe Verbindung, das queck silbersulfonsaure Natrium, das schon früher von $\mathrm{B}$ a $\mathrm{r} \mathrm{th}^{\mathbf{4}}$ ) auf anderem Wege, nämlich durch Auflösen von Quecksilberoxyd in Natriumbisulfitlösung, erhalten worden war.

Bezüglich der Einwirkung von Quecksilberchlorid auf Thiosulfate und Polythionate hingegen schloß ich mich der von $\mathrm{Fel} \mathrm{d}^{5}$ ) geäußerten Ansicht an, daß das Quecksilberchlorid auf diese Salze oxydierend wirke, und zwar nach folgenden Gleichungen:

1) Vgl. Angew. Chem. 28, I, 9 und 273 [1915].

2) Angew. Chem. 28, I, 9 [1915].

3) Chem. Industr. 21, 378 [1898].

4) Z. physik. Chem. 9, 192 [1892].

5) Angew. Chem. 24, 293 [1911].

$$
\text { (2) } \begin{aligned}
\mathrm{Na}_{2} \mathrm{~S}_{2} \mathrm{O}_{3}+2 \mathrm{HgCl}_{2}+\mathrm{H}_{2} \mathrm{O}=\mathrm{Na}_{2} \mathrm{SO}_{4}+\mathrm{Hg}_{2} \mathrm{Cl}_{2} \\
+2 \mathrm{HCl}+\mathrm{S} \\
\text { (3) } \quad \mathrm{Na}_{2} \mathrm{~S}_{3} \mathrm{O}_{6}+2 \mathrm{HgCl}_{2}+2 \mathrm{H}_{2} \mathrm{O}=\mathrm{Na}_{2} \mathrm{SO}_{4}+\mathrm{Hg}_{2} \mathrm{Cl}_{2} \\
+2 \mathrm{HCl}+\mathrm{H}_{2} \mathrm{SO}_{4}+\mathrm{S} . \\
\text { (4) } \quad \mathrm{Na}_{2} \mathrm{~S}_{4} \mathrm{O}_{3}+2 \mathrm{HgCl}_{2}+2 \mathrm{H}_{2} \mathrm{O}=\mathrm{Na}_{2} \mathrm{SO}_{4}+\mathrm{Hg}_{2} \mathrm{Cl}_{2} \\
+2 \mathrm{HCl}+\mathrm{H}_{2} \mathrm{SO}_{4}+2 \mathrm{~S} .
\end{aligned}
$$

Wenn ich diese Umsetzungsgleichungen als richtig annahm, so geschah es deshalb, weil die Titration der gebildeten freien Säure tatsächlich die durch obige Gleichungen ausgedrückten Werte ergab, nämlich auf $1 \mathrm{Mol}$. Thiosulfat 2 Aquivalente und auf 1 Mol. Polythionat 4 Äquivalente freie Säure. Bei näherem Studium dieser Reaktionen kamen mir jedoch einige Bedenken; vor allem ist nicht recht einzusehen, warum Thiosulfate und Polythionate mit wenig Quecksilberchlorid so ganz anders reagieren sollen als mit einem Ưberschuß von Quecksilberchlorid. Feld gibt nämlich an, daß die Umsetzung nach Gleichung 2, 3 und 4 nur bei Überschuß von Quecksilberchlorid vor sich geht, wogegen mit wenig Quecksilberchlorid die Reaktionen nach folgenden Gleichungen verlaufen sollen:

(5) $\mathrm{Na}_{2} \mathrm{~S}_{2} \mathrm{O}_{3}+\mathrm{HgCl}_{2}+\mathrm{H}_{2} \mathrm{O}=\mathrm{Na}_{2} \mathrm{SO}_{4}+\mathrm{HgS}+2 \mathrm{HCl}$.

(6) $\mathrm{Na}_{2} \mathrm{~S}_{3} \mathrm{O}_{6}+\mathrm{HgCl}_{2}+2 \mathrm{H}_{2} \mathrm{O}=\mathrm{Na}_{2} \mathrm{SO}_{4}+\mathrm{HgS}+2 \mathrm{HCl}$

$$
+\mathrm{H}_{2} \mathrm{SO}_{4} \text {. }
$$

(7) $\mathrm{Na}_{2} \mathrm{~S}_{4} \mathrm{O}_{6}+\mathrm{HgCl}_{2}+2 \mathrm{H}_{2} \mathrm{O}=\mathrm{Na}_{2} \mathrm{SO}_{4}+\mathrm{HgS}+2 \mathrm{HCl}$

$$
+\mathrm{H}_{2} \mathrm{SO}_{4}+\mathrm{S} \text {. }
$$

In diesem Falle erhält man also statt eines weißen Niederschlages einen schwarzen, nämlich Quecksilbersulfid; ferner wird bei dem Thiosulfat und dem Trithionat kein freier Schwefel abgeschieden, sondern nur bei dem Tetrathionat, und zwar nur ein Atom Schwefel statt der zwei Atome, die die Gleichung 4 verlangt. Die Menge der gebildeten freien Säure ist dagegen in den Gleichungen 5,6 und 7 die nämliche wie in den Gleichungen 2, 3 und 4.

Unter Berücksichtigung der schon ziemlich weit zurückliegenden Untersuchungen von $\mathrm{Her} \mathrm{s} \mathrm{ch}$ el, $\mathrm{R}$ o s e und von $K$ e $B$ l e $r$ sowie der ganz ähnlichen Reaktion zwischen Thiosulfat und Silbernitrat kommt man zu dem Resultat, claß die fraglichen Reaktionen nach den Gleichungen 5, 6 und 7 vor sich gehen müssen, während die von $\mathrm{Fel} \mathrm{d}$ aufgestellten Gleichungen 2, 3 und 4 den Vorgang nicht richtig wiedergeben. Allerdings ist zu bemerken, daß auch die Gleichungen 5, 6 und 7 nur den Endzustand einer verwickelteren Umsetzung darstellen. Die Erscheinung, daß man bei Anwendung von überschüssigem Quecksilberchlorid keinen schwarzen, sondern einen weißen Niederschlag erhält, läßt sich ferner in sehr einfacher Weise erklären, da das frisch gefällte Quecksilbersulfid bekanntlich auf Quecksilberchlorid unter Bildung eines weißen, in Wasser unlöslichen Sulfochlorids einwirkt:

$$
2 \mathrm{HgS}+\mathrm{HgCl}_{2}=\mathrm{Hg}_{3} \mathrm{~S}_{2} \mathrm{Cl}_{2} \text {. }
$$

Ehe ich für die Richtigkeit dieser Behauptung den experimentellen Nachweis erbringe, sollen kurz einige frühere Untersuchungen hier angeführt werden, deren Verfasser zum Teil zu demselben Ergebnis gelangten.

Der erste, der die Einwirkung von Quecksilberchlorid

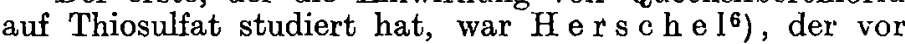
fast 100 Jahren beim Versetzen einer verd. Lösung von Calciumthiosulfat mit viel Quecksilberchlorid die Bildung eines gelblichen Niederschlages beobachtete. Diesen Niederschlag hielt er für ein Gemisch von Quecksilberthiosulfat, Kalomel und Schwefel, zugleich bemerkte er, daß die überstehende Flüssigkeit sauer reagierte, und glaubte, daß sie freie Schwefelsäure enthalte. Im Jahre 1834 hat dann $\mathrm{H} . \mathrm{R}$ o s $\mathrm{e}^{7}$ ) diese Versuche wiederholt und gezeigt, daß der Niederschlag kein Quecksilberthiosulfat enthalten kann, da dieses Salz sehr unbeständig ist und im Moment seiner Entstehung zerfällt. Er erklärte den Vorgang folgendermaßen: „Setzt man eine geringe Menge Quecksilberchlorid zu einem t'berschuß von Thiosulfat, so entsteht zwar ein weißer Niederschlag, der indessen sogleich gelb, braun und endlich durch längeres Stehen, oder schneller durch Kochen,

6) Edinburgh Philos. Journ. 1, 28 [1819].

7) Pogg. Ann. 33, 240 [1834]. 
schwarz wird. Der entstandene schwarze Niederschlag ist Quecksilbersulfid; die über ihm stehende Flüssigkeit enthält Schwefelsäure. Vermischt man hingegen einen großen Überschuß von Quecksilberchlorid mit einer geringen Menge Thiosulfat, so entsteht sogleich ein weißer Niederschlag, der lange in der Flüssigkeit suspendiert bleibt und weder durch langes Stehen, noch durch Kochen seine Farbe verändert. Er besteht aus einer unlöslichen Verbindung von Quecksilbersulfid mit Quecksilberoxyd, die von ihm getrennte Flüssigkeit enthält Schwefelsäure. Der schwarze Quecksilbersulfidniederschlag wird, wenn er mit Quecksilberchlorid geschüttelt wird, wiederum weiß, indem sich die erwähnte Verbindung bildet . .."

Wie man hieraus ersieht, hat $R$ o s e den Reaktionsverlauf in der Hauptsache schon richtig erkannt, eine Reaktionsgleichung hat jedoch erst $K$ e $B l$ e $r^{8}$ ) aufgestellt, der namentlich auch die Umsetzung der Polythionate mit Quecksilberchlorid und-cyanid näher studiert hat. Er sagt: ,Quecksilberchlorid gibt mit Trithionsäure eine weiße, mit Tetra- und Pentathionsäure eine gelbliche Fällung. Diese Niederschläge sind die bekannten V e r bindungen von Quecksilbersulfid mit Quecksilberchlorid und enthalten bei der Tetraund Pentathionsäure noch $f \mathrm{rei}$ en $\mathrm{S}$ ch wef e l".

Zur Klärung der Widersprüche, die zwischen diesen älteren Untersuchungen und den Angaben F e l d s bestehen, hielt ich es für zweckmäßig, durch quantitative Bestimmung der entstehenden Quecksilberniederschläge den wirklichen Reaktionsverlauf festzustellen. Dies kann in sehr einfacher Weise geschehen, da das Gewicht der Niederschläge sehr verschieden ist, je nachdem die Umsetzung nach der von $\mathrm{K}$ e $\mathrm{Bl}$ e $\mathrm{r}$ oder der von $\mathrm{F}$ eld angenommenen Gleichung verläuft. Die Bestimmung wurde mit Thiosulfat, Trithionat und Tetrathionat ausgeführt, und zwar in der Weise, da $B$ eine abgewogene Menge der Schwefelverbindung in Wasser gelöst und in der Wärme mit überschüssigem Quecksilberchlorid versetzt wurde. Nach mehrstündigem Stehen wurde der Niederschlag auf ein gewogenes Filter gebracht und bei $100^{\circ}$ bis zur Gewichtskonstanz getrocknet. Das Ergebnis war folgendes:

a) Natriumthiosulfat: Nach Feld verläuft die Umsetzung nach der obigen Gleichung $2, \mathrm{~d}$. h. man erhält auf $1 \mathrm{Mol}$. Thiosulfat einen Niederschlag, der aus $1 \mathrm{Mol}$ Mercurochlorid und 1 Atom Schwefel besteht. Nach der wahrscheinlicheren Anmahme von $K$ e B l e r verläuft die Umsetzung hingegen nach der Gleichung:

(9) $2 \mathrm{Na}_{2} \mathrm{~S}_{2} \mathrm{O}_{3}+3 \mathrm{HgCl}_{2}+2 \mathrm{H}_{2} \mathrm{O}=\mathrm{Hg}_{3} \mathrm{~S}_{2} \mathrm{Cl}_{2}+4 \mathrm{NaCl}$

Man erhält in diesem Falle also auf $2 \mathrm{Mol}$. Thiosulfat 1 Mol. der komplexen Verbindung $\mathrm{Hg}_{3} \mathrm{~S}_{2} \mathrm{Cl}_{2}$, aber k e i n e n freien Schwefel. Es wurden angewandt 2,5 g $\mathrm{Na}_{2} \mathrm{~S}_{2} \mathrm{O}_{3}$ (=10 Millimol) und $5,4 \mathrm{~g} \mathrm{HgCl}_{2}(=20 \mathrm{Millimol})$. Bein Zusammengießen der beiden warmen Lösungen entstand zuerst ein schwarzer Niederschlag, der jedoch alsbald heller und schließlich gelblichweiß wurde. Der Niederschlag wurde auf dem Filter so lange ausgewaschen, bis die ablaufende Flüssigkeit nicht mehr sauer reagierte. Das Gewicht des Niederschlags berechnet sich nach F e l d (Gleichung 2) zu $5,03 \mathrm{~g}$, nach $\mathrm{K}$ e $\$ \mathrm{l}$ e $\mathrm{r}$ (Gleichung 9) dagegen zu 3,67 g. Es wurde gefunden: $3,60 \mathrm{~g}$.

b) Kali um trithionat: Die Umsetzung des Trithionats mit Quecksilberchlorid soll nach $\mathbf{F}$ e l d den gleichen Niederschlag $\left(\mathrm{Hg}_{2} \mathrm{Cl}_{2}+\mathrm{S}\right)$ ergeben wie das Thiosulfat (Gleichung 3). Nach K e B l e r erhält man indessen wieder die komplexe Verbindung $\mathrm{Hg}_{3} \mathrm{~S}_{2} \mathrm{Cl}_{2}$, entsprechend der Gleichung:

(10) $2 \mathrm{~K}_{2} \mathrm{~S}_{3} \mathrm{O}_{6}+3 \mathrm{HgCl}_{2}+4 \mathrm{H}_{2} \mathrm{O}=\mathrm{Hg}_{3} \mathrm{~S}_{2} \mathrm{Cl}_{2}+4 \mathrm{KCl}$

$$
+4 \mathrm{H}_{2} \mathrm{SO}_{4} \text {. }
$$

Die Umsetzung der beiden Salze wurde in gleicher Weise ausgeführt wie beim Thiosulfat. Es wurden angewandt $2,7 \mathrm{~g}$ (=10 Millimol) $\mathrm{K}_{2} \mathrm{~S}_{3} \mathrm{O}_{6}$ und $5,4 \mathrm{~g}$ (=20 Millimol) $\mathrm{HgCl}_{2}$. Beim Zusammengießen der Lösungen entstand ein weißer Niederschlag. Sein Gewicht berechnet sich nach

8) J. prakt. Chem. 4\%, 42 [1849].
$\mathrm{F}$ e $\mathrm{l}$ d (Gleichung 3 ) zu $5,03 \mathrm{~g}$, nach $\mathrm{K}$ e $ß \mathrm{l}$ e $\mathrm{r}$ (Gleichung 10 ) $\mathrm{zu} 3,67 \mathrm{~g}$. Es wurde gefunden: $3,65 \mathrm{~g}$.

c) Ka liumtetrathionat: Hier soll nach Feld ein aus $\left(\mathrm{Hg}_{2} \mathrm{Cl}_{2}+2 \mathrm{~S}\right)$ bestehender Niederschlag (Geichung 4) gebildet werden, während nach $\mathrm{K}$ e $B \mathrm{l}$ e r die Umsetzung nach folgender Gleichung verläuft:

$$
\text { (11) } \begin{aligned}
2 \mathrm{~K}_{2} \mathrm{~S}_{4} \mathrm{O}_{6} & +3 \mathrm{HgCl}_{2}+4 \mathrm{H}_{2} \mathrm{O}=\mathrm{Hg}_{3} \mathrm{~S}_{2} \mathrm{Cl}_{2}+2 \mathrm{~S} \\
+ & 4 \mathrm{H}_{2} \mathrm{SO}_{4}+4 \mathrm{KCl} .
\end{aligned}
$$

Der Niederschlag enthält also hier im Gegensatz zu den bisher besprochenen Umsetzungen neben der komplexen Verbindung $\mathrm{Hg}_{3} \mathrm{~S}_{2} \mathrm{Cl}_{2}$ noch freien Schwefel, und zwar auf $1 \mathrm{Mol} . \mathrm{K}_{2} \mathrm{~S}_{4} \mathrm{O}_{6}$ nur 1 Atom $\mathrm{S}$. Es wurden angewandt $1,5 \mathrm{~g}$ (=5 Millimol) $\mathrm{K}_{2} \mathrm{~S}_{4} \mathrm{O}_{8}$ und $2,7 \mathrm{~g}\left(=10\right.$ Millimol) $\mathrm{HgCl}_{2}$, der Niederschlag war gelblich gefärbt. Sein Gewicht berechnet sich nach $\mathrm{F}$ eld (Gleichung 4) zu 2,67 g, nach $\mathrm{Ke} B \mathrm{le} \mathrm{r}$ (Gleichung 11) zu 2,0 g. Es wurde gefunden: 2,02 g. Hiervon wurden $1,94 \mathrm{~g}$ mit Schwefelkohlenstoff extrahiert, wobei der von $\mathrm{Fr}$ i ed $\mathrm{h}$ e i $\mathrm{m}$ und $\mathrm{M}$ i $\mathrm{c} \mathrm{h}$ a e I i $\mathrm{s}^{9}$ ) angegebene Apparat benutzt wurde. Der Gewichtsverlust betrug $0,15 \mathrm{~g} \mathrm{~S}$ (berechnet $0,155 \mathrm{~g}$ ).

Die vorstehenden drei Analysen zeigen mit voller Deutlichkeit, daß Quecksilberchlorid auf Thiosulfat und Polythionate $\mathrm{nicht}$ oxydierend wirkt, wie Feld annahm, sondern daß hierbei einfach eine doppelte Umsetzung stattfindet, wie Ke B ler bereits vor 67 Jahren richtig beobachtet hat. Das hierbei gebildete Quecksilberthiosulfat bzw. -polythionat ist jedoch sehr unbeständig und zerfällt sofort in Quecksilbersulfid und freie Schwefelsäure (bei Tetrathionat entsteht auch noch freier Schwefel), worauf sich das Quecksilbersulfid mit dem überschüssigen Quecksilberchlorid zu dem komplexen Salz $\mathrm{Hg}_{3} \mathrm{~S}_{2} \mathrm{Cl}_{2}$ verbindet. Die Reaktion verläuft also in folgenden drei Phasen:

a) bei dem Natriumthiosulfat:

(12) 1. $\mathrm{Na}_{2} \mathrm{~S}_{2} \mathrm{O}_{3}+\mathrm{HgCl}_{2}=\mathrm{HgS}_{2} \mathrm{O}_{3}+2 \mathrm{NaCl}$.

2. $\mathrm{HgS}_{2} \mathrm{O}_{3}+\mathrm{H}_{2} \mathrm{O}=\mathrm{HgS}+\mathrm{H}_{2} \mathrm{SO}_{4}$.

3. $2 \mathrm{HgS}+\mathrm{HgCl}_{2}=\mathrm{Hg}_{3} \mathrm{~S}_{2} \mathrm{Cl}_{2}$.

b) bei dem Kaliumtrithionat:

(13) I. $\mathrm{K}_{2} \mathrm{~S}_{3} \mathrm{O}_{6}+\mathrm{HgCl}_{2}=\mathrm{HgS}_{3} \mathrm{O}_{6}+2 \mathrm{KCl}$.

2. $\mathrm{HgS}_{3} \mathrm{O}_{6}+2 \mathrm{H}_{2} \mathrm{O}=\mathrm{HgS}+2 \mathrm{H}_{2} \mathrm{SO}_{4}$

3. $2 \mathrm{HgS}+\mathrm{HgCl}_{2}=\mathrm{Hg}_{3} \mathrm{~S}_{2} \mathrm{Cl}_{2}$.

c) bei dem Kaliumtetrathionat:

(14) 1. $\mathrm{K}_{2} \mathrm{~S}_{4} \mathrm{O}_{6}+\mathrm{HgCl}_{2}=\mathrm{HgS}_{4} \mathrm{O}_{6}+2 \mathrm{KCl}$.

2. $\mathrm{HgS}_{4} \mathrm{O}_{6}+2 \mathrm{H}_{2} \mathrm{O}=\mathrm{HgS}+2 \mathrm{H}_{2} \mathrm{SO}_{4}+\mathrm{S}$.

3. $2 \mathrm{HgS}+\mathrm{HgCl}_{2}=\mathrm{Hg}_{3} \mathrm{~S}_{2} \mathrm{Cl}_{2}$.

Man darf ohne weiteres annehmen, daß die Unisetzung bei dem Pentathionat in genau der gleichen Weise verläuft, nur erhält man hier ein Atom freien Schwefel mehr als bei dem Tetrathionat. Die von F e l d aufgestellten Reaktionsgleichungen (Gleichung 2, 3 und 4) sind demnach nicht zutreffend, vielmehr bildet die Umsetzung des Quecksilberchlorids mit Thiosulfat und Polythionaten ein vollkommenes Analogon zu der von $\mathrm{Her} \mathrm{s} \mathrm{ch} \mathrm{e} \mathrm{l}^{10}$ ) zuerst beobachteten und später von $G$ e $u t h$ e $\mathbf{r}^{11}$ ) näher untersuchten Einwirkung von Silbernitrat auf Thiosulfat, wobei als Endprodukt Schwefelsilber und ebenfalls freie Schwefelsäure entstehen. Auch diese Umsetzung verläuft quantitativ und kann, wie $\mathrm{B}$ o d n á $\mathrm{r}^{12}$ ) erst kürzlich gezeigt hat, zur titrimetrischen Bestimmung von Thiosulfat neben Sulfit Anwendung finden.

Schließlich sei noch darauf hingewiesen, daß auch Umsetzung von Alkalisulfiden mit überschüssigem Quecksilberchlorid, wie bekannt, in ganz analoger Weise verläuft. Auch hier entsteht die komplexe Verbindung $\mathrm{Hg}_{3} \mathrm{Cl}_{2} \mathrm{~S}_{2}$, daneben aber $\mathrm{k}$ e i $\mathrm{n}$ e freie Säure. Auf der Grundlage dieser Reaktion habe ich eine neue Methode zur Bestimmung von Sulfiden, Sulfiten und Thiosulfaten nebeneinander ausgearbeitet, über die an anderer Stelle ${ }^{13}$ ) vor kurzem ausführlich berichtet wurde. (SchluB folgt.)

9) Tread we 11, Lehrbuch, 4. Aufl.; Bd. II, 124.

10) Edinburgh Philos. Journ. 1, 16, 18, 397 [1819].

11) Liebigs Ann. 226, 232 [1884]. $[19147$.

12) Z. anal. Chem. 53, 37-41 [1914]; Angew. Chem. 27, II, 266

13) Chem.-Ztg. 39, 945 [1915] 